\title{
Variations
}

Variations

Revue internationale de théorie critique

16 | 2012

Tahrir is here !

\section{La production numérique du réel, perspectives anthropologiques}

\section{Monique Selim}

\section{(2) OpenEdition}

\section{Journals}

\section{Édition électronique}

URL : http://journals.openedition.org/variations/148

DOI : 10.4000/variations. 148

ISSN : 1968-3960

\section{Éditeur}

Les amis de Variations

\section{Édition imprimée}

Date de publication : 16 janvier 2012

\section{Référence électronique}

Monique Selim, «La production numérique du réel, perspectives anthropologiques », Variations [En ligne], 16 | 2012, mis en ligne le 20 février 2012, consulté le 20 avril 2019. URL : http:// journals.openedition.org/variations/148 ; DOI : 10.4000/variations.148

Ce document a été généré automatiquement le 20 avril 2019.

Les ami•e•s de Variations 


\title{
La production numérique du réel, perspectives anthropologiques
}

\author{
Monique Selim
}

\section{NOTE DE L'ÉDITEUR}

Première publication sur www.theoriecritique.com, « Tahrir is here ! ", 16 janvier 2012, pp. 106-113

Toutes les sociétés et les Etats sont désormais concernés par Internet, tout comme les différentes instances sociales, politiques, économiques et scientifiques aux échelles nationales et globales : condition de possibilité des échanges de toute nature, Internet, par l'ouverture extraordinaire qu'il offre est aussi une menace pour les gouvernements autoritaires, parmi lesquels certains envisagent de créer un Intranet propre à leurs pays ou alors développent comme en Chine des Blogs de fonctionnaires supposés assurer le contrôle des populations par une pseudodémocratie instantanée. Les dossiers médiatiques et les études sont innombrables et chaque discipline de sciences sociale a mis en œuvre une prise en compte spécifique de l'outil numérique, en accord avec son appareil méthodologique. Ainsi, pour les sciences politiques l'articulation des mouvements sociaux en Tunisie, en Egypte, en Libye en Syrie, en 2010-2011 - qu'on qualifie de "révolutionnaires » - avec la diffusion numérique des informations, s'impose maintenant comme un questionnement important nécessitant une démarche nouvelle. En anthropologie on a assisté à la création d'une branche propre qui revêt divers noms : digital anthropology, cyberanthropologie, anthropologie numérique, etc. Cette opération de division et de spécialisation selon la nature des terrains (religion, économie, éducation, développement, genre, etc.) est historiquement classique dans la discipline et présente des dimensions plus ou moins positives et négatives selon l'objet retenu et sa conceptualisation. Néanmoins, dans ce cadre de segmentation et de développement disciplinaires, Internet mérite une attention toute particulière car ont émergé majoritairement des investigations centrées exclusivement sur la toile, focalisées sur 
divers supports et leurs appropriations par telle ou telle catégorie d'acteurs. Celles-ci s'efforcent d'élaborer une approche adéquate dont la légitimation se joue dans la confrontation avec les terrains sociaux usuels. Il s'agit principalement de faire rentrer et accepter Internet dans la liste des terrains de recherche anthropologique et de démontrer que les techniques habituelles peuvent y être appliquées: l'observation participante, l'entretien principalement. Stéphane Heas et Véronique Poutrain (2003) décrivent ainsi "les méthodes d'enquête qualitative sur Internet ", soulignant "qu'Internet apparaît comme un terrain semblable aux autres... », " qu'il se présente au chercheur telle une ville qu'il ne connaît pas et qu'il va apprendre à découvrir ». Cette métaphore rappelle les débats de la fin des années 70 - alors même que l'anthropologie urbaine faisait une irruption provocatrice dans la discipline - sur la ville. Notons qu'avec la banalisation de l'urbain dans la discipline, la question s'est d'elle-même évaporée. Pour beaucoup, cependant, il incomberait à l'anthropologue face à Internet, de gérer comme dans d'autres situations d'enquête, les biais inévitables qui se sont infiltrés dans son travail. Pour féconds, que se manifestent déjà ces nombreux travaux existants effectués dans cette optique, ils pâtissent pourtant souvent de l'autonomisation de la sphère numérique qu'ils inscrivent dans la construction même de leur objet et de la «tentation ethnographique » qu'ils lui dédient. Holiste par vocation l'anthropologie se différencie des autres sciences sociales par l'appréhension des articulations des différents champs sociaux dans lesquels les individus se meuvent et du sens qu'ils donnent à leurs actions. La cohérence des logiques subjectives et objectives intriquées se dévoile dans l'effort de retotalisation de l'anthropologue, impliqué dans les relations interpersonnelles et faisant de cette implication un outil épistémologique. Internet serait-il un champ social exceptionnel, et/ou supplémentaire qui s'offrirait au regard anthropologique? Comment faudrait-il alors traiter ce supplément de nature inédite? Deux voies s'offrent au chercheur comme pour tout champ social : l'approfondissement par sectionnement et isolement - très privilégié aujourd'hui - ou le replacement dans un contexte complexe, plus large à décortiquer, orientation que nous tenterons ici d'argumenter.

2 Plutôt que d'affranchir Internet en le constituant en terrain en quelque sorte autosuffisant à lui-même, l'hypothèse semble plus heuristique, dans un mouvement inverse, de l'intégrer à toute enquête anthropologique menée aujourd'hui, dans la mesure précisément où il innerve l'ensemble de la quotidienneté des acteurs dans leur intimité autant que dans leurs différentes sphères d'insertion collective. Cette diffusion numérique omniprésente dans le travail, la parenté, le politique, le religieux - grands pivots de toute investigation - provoquent des transformations importantes, en termes de méthodes, de concepts, d'objets dans les recherches anthropologiques et ce, y compris là où une minorité de gens est reliée à Internet. Il suffit pour s'en convaincre de prendre l'exemple des marchés matrimoniaux, tout à la fois globalisés par Internet et aux endogamies resserrées par des logiques identitaires et ethnicisées qui profitent de l'outil numérique pour s'intensifier.

\section{Dissolutions et recréations catégorielles}

3 Internet oblige tout d'abord l'anthropologue à des remaniements catégoriels, des lors qu'est abandonné le vocable simplificateur de virtuel, qui pousse à l'autonomisation de mondes dits virtuels, dont la place en regard du réel et de l'imaginaire reste indécise. Le numérique habite le réel autant que l'imaginaire et contribue à la production de 
nouvelles réalités comme d'imaginaires inédits aux différentes échelles qui partent de l'individu singulier jusqu'au monde global auquel Internet a donné forme en édifiant la possibilité d'une sphère globalisée de communication et en lui donnant corps. Symbolique, imaginaire ou réel - pour reprendre une trilogie classique - le lien social inclut maintenant une dimension numérique, qui n'a rien de "virtuelle» mais se déchiffre comme ses autres attributs dans les relations mutuelles inscrites entre les trois sphères.

4 En revanche, c'est bien la rupture entre vie privée et vie publique qui s'effondre avec la numérisation des échanges. Constitutive de l'analyse anthropologique, mais aussi sociologique, cette rupture était à la base d'interprétations qui paraissaient solides et durables dans les sociétés industrialisées, alors opposées à des univers lointains parés de l'immédiateté des relations et œuvrant en faveur de l'inexistence de l'individu fondu dans le collectif. Dans ces dualisations emboitées il semblait que « ici » la famille était le lieu du privé, conçu comme un refuge face aux oppressions de la société extérieure et en particulier face au monde du travail. En miroir on imaginait qu' « ailleurs » le privé était inexistant dans le contexte d'une sorte de plénitude sociale. Ces constructions en écho, fortement ethnocentristes semblent d'autant plus obsolètes qu'Internet omniprésent partout est l'un des instruments les plus percutants dans l'abolition du «Nord» et des "Suds», d'ici et d'ailleurs, de sociétés «industrielles» et d'autres rurales. Plus précisément la médiation que réalise la sphère numérique dans les relations interpersonnelles et par exemple familiales donne à penser un des aspects de l'inutilité actuelle de la dichotomie privé/public: que des parents s'inscrivent sur Facebook pour "contrôler" les fréquentations de leurs enfants qui affichent sur le site pensées et évènements, sans distinction d'une illusoire intimité donnée au regard de tous, met en scène avec force l'infiltration numérique des modes de communication depuis leur niveau le plus restreint jusqu'à leur expansion globale évidente. De ce point de vue, les accusations de non-protection de la "vie privée " qui de façon régulière viennent dénoncer les intrusions d'Internet, paraissent des résidus de l'ancienne rupture entre vie privée et vie publique. La surproduction de la réalité qu'initie l'outil numérique de par son incommensurable puissance de démultiplication instantanée et d'intensification spectaculaire réduit les sursauts de défense de la "vie privée » en symptômes d'une communication régulée, mais défunte. A un autre niveau, l'ostentation abyssale de soi que permet Internet, a travers des sites individuels - dont l'usage s'est répandu dans toutes les couches de la population (dont les chercheurs) avec des significations différentes renforce une logique qui, de fait, agglutine dans l'indistinction réel, imaginaire et symbolique en une nouvelle entité englobante, fascinante, pétrifiant individus et collectifs dans une sorte de titrisation narcissique infinie.

5 Avec la dissolution de la division entre privé/public au profit d'une visibilisation exponentielle de sois individuels et de collectifs de toute nature, c'est une seconde notion fondamentale pour l'anthropologie qui demande à être revisitée : celle de groupe social, conçu dans notre discipline comme l'échelon micro exemplaire de la démarche anthropologique: l'anthropologue s'immerge dans un groupe social dont il appréhende tous les contours et en devenant un acteur de la communication interpersonnelle qui le caractérise, il en saisit les clefs d'organisation et de fonctionnement s'efforçant dans un second temps, de mettre en évidence la représentativité du groupe dans un contexte sociétal plus large. Depuis l'origine de l'anthropologie, ce groupe social est par définition concret et l'originalité de la démarche tient précisément à cette dimension tangible de 
l'interconnaissance dont s'empare l'anthropologue. Entendue de cette façon - qui puise au départ dans l'expérience des anthropologues découvrant les communautés rurales lointaines dans lesquelles tous les champs sociaux, parenté, politique, religion, sont confondus dans une seule unité territoriale, de surcroît artificiellement figée dans l'atemporalité - la notion de groupe social achève sont éclatement. Rappelons en effet que les recherches sur les migrations ont dans les 20 dernières années déjà largement entamé sa pertinence en mettant en évidence la déterritorialisaiton constitutive de groupes présents à la fois sur de multiples lieux - conçus d'abord comme duels ici et là bas, dans le topos originaire, et bientôt se développant sur de multiples scènes en étroite relation les unes avec les autres au plan économique, politique, religieux et matrimonial, au point de figurer des reterritorialisations imaginaires d'où l'expression innovante d'une anthropologie multisites. Internet, non seulement accompagne et fortifie cet essaimage des groupes, mais surtout impose de concevoir l'existence de groupes sociaux que je définirais comme abstraits en regard des groupes concrets étudiés historiquement dans la discipline anthropologique. Nullement virtuels, comme on a coutume de le penser, ces groupes sociaux abstraits émergent, prennent leur élan et puisent leur force dans la sphère globale de communication où ils font leur entrée et tentent au sens propre d'exister. Ces groupes sociaux abstraits - véritables symptômes et acteurs idéologiques d'une globalisation à laquelle la numérisation des univers est consubstantielle, ont des ancrages les plus divers qui seuls ou combinés offrent des paysages d'une très grande richesse. La dissidence politique nationale associée ou non à l'appartenance ethnoculturelle et arrimée de façon plus ou moins bien bricolée aux thématiques de la gouvernance démocratique et des droits de l'homme en constitue un bon exemple que l'on retrouve de l'Ouzbékistan (Dilorom Usmanova 2010), à la Chine, y compris dans les minorités nationales de l'empire, chez les homosexuels (Wenjing Guo 2011), comme dans les bourgeonnements féministes (Selim 2011). L'affiliation religieuse, la revendication d'une origine commune débouchant sur une identité partagée reconnue et aspirant à une construction normative et défensive comme pour les expatriés du Kirghiztan (Ulugbek Badalov 2011) en propose une autre version. Un événement, un individu, une cause sont des enjeux de mobilisation numérique qui s'inscrivent quotidiennement sur la sphère de communication globalisée que dessine la toile. Le tremblement de terre au Setchuan en 2007 a vu ainsi éclore deux types de groupes: les volontaires spontanés venus se précipiter pour sauver les sinistrés et suivant l'une ou l'autre des multiples initiatives individuelles sur la Toile qui ont embarrassé fortement les secours officiels, les repoussant; les parents découvrant la corruption des fonctionnaires locaux ayant empoché les financements des écoles, s'effondrant des lors comme du "fromage de soja ", selon la métaphore employée et décidés à affronter le gouvernement au niveau provincial ou national. Encore en Chine, l'éducation des enfants des campagnes se présente comme un formidable ressort de formation de groupes de jeunes partant aider les enseignants ruraux dans leurs tâches. Dans tous les cas, ces groupes abstraits initient une interconnaissance idéelle et la question pour l'anthropologue se déploie en deux volets: ce groupe abstrait va-t-il se satisfaire d'une communication immatérielle et n'inscrire son existence que sur le marché numérique spécifique où il se situe, se consacrant alors à se maintenir dans une âpre concurrence, ou bien le groupe abstrait va-t-il déboucher sur une actualisation concrète et une action dans la réalité. Dans le premier cas, la démarche la plus fréquemment mise en œuvre recommande une plongée dans l'univers numérique singulier retenu avec éventuellement des entretiens sur la Toile. Ceux-ci, cependant, ne permettent pas de replacer les acteurs dans leur propre constellation de rapports sociaux 
et d'inscriptions personnelles, alors qu'il faudrait précisément comprendre dans une perspective anthropologique les modes d'accrochage au réel qu'instituent ces insertions individuelles dans des interconnaissances idéelles ce qui suppose des enquêtes partant des personnes elles-mêmes. Dans le second cas il est encore plus clair que seules des investigations sur les dynamiques concrètes engendrées par le groupe à l'origine abstrait peuvent permettre de saisir le sens des articulations en jeu entre les différentes sphères d'investissement des acteurs individuels et collectifs. La difficulté principale pour l'anthropologue réside dans la nécessité de suivre dans leur cheminement et leur investissement personnel les individus de toutes provenances qui ont rejoint le groupe numérique et y sont actifs. Les logiques individuelles mettent en scène des palettes extrêmement diverses de relations entre le réel, l'imaginaire et le symbolique et l'anthropologue découvre là des modalités existentielles en formation indiscernables avec l'enfermement de la recherche dans l'univers numérique, à la manière des études sur la sexualité et les rencontres amoureuses à Hong Kong (Pei Yuxin 2007, Ding Yu 2009, Yangwen 2009).

\section{Positionnements méthodologiques}

6 Groupe social abstrait, interconnaissance idéelle, espace globalisé de communication ont été proposés comme des repères conceptuels embryonnaires pour avancer au plan épistémologique dans une anthropologie du contemporain qui intègre pleinement l'outil numérique, ses effectuations et ses actualisations de natures très contrastées. Néanmoins ne semblent pas remises en cause les catégories de l'imaginaire, du symbolique et du réel, qui au contraire d'une certaine manière se voient conférer plus d'amplitude dans leurs interconnexions. Internet offre en effet des surfaces de déploiement extraordinaires aux imaginaires individuels et collectifs, de même qu'il démultiplie les capacités de reformulation du capital symbolique des sociétés et l'efficacité symbolique des pratiques et des discours. D'une manière générale c'est l'ensemble des modes d'investissement symbolique et imaginaire qui trouvent dans l'outil numérique un nouvel envol.

7 En revanche, comme à chaque étape de l'évolution de la discipline, la position de l'anthropologue se trouve interpellée. Dans la période qui a suivi les décolonisations, le rapatriement des anthropologues sur leur société d'appartenance a ainsi provoqué une première série de réflexions : la distance dite culturelle, la posture d'étranger, dont la dimension de domination politico-économique était majoritairement occultée, avaient en effet été théorisées comme des prédicats épistémologiques de la condition intellectuelle de l'anthropologue. S'aventurer à mener des investigations dans sa propre société revenait pour beaucoup à devenir sociologues, destinée perçue comme une sorte de chute. Que l'anthropologue pouvait-il bien faire dans des sociétés que le structuralisme avait définitivement rangées dans l'anonymat et l'individualisme? Une réponse fut trouvée dans une reproduction fabuleuse des dispositifs « exotiques » au sein même de la société de l'anthropologue : reconstruire la distance culturelle avec les groupes étudiés des ouvriers aux bureaucrates, des employés hospitaliers aux SDF, etc. - réédifier l'autonomie de ces groupes constitués en étrangers au monde propre de l'anthropologue, s'y immerger comme s'il s'agissait de communautés aux mœurs tribales singulières, aux termes d'adresses inconnus, aux rituels inédits, bref en «faire une ethnographie » avec des « informateurs », indispensables attributs de légitimation de l'opération. 
8 La numérisation des univers quotidiens et du monde global n'offre pas les conditions de possibilité d'une telle procédure de mime pour asseoir actuellement la connaissance anthropologique. La première raison en est sans aucun doute la percée de l'outil numérique dans les univers professionnels de la recherche. Comme dans toutes les disciplines, l'anthropologue, à toutes les phases de son travail, est relié par Internet à son/ses terrains, ses collègues, son institut de rattachement, et bien sûr les banques de données qui lui apportent les informations indispensables. Continuellement joignable par l'une ou l'autre des instances qui étayent son existence, évalué au plan personnel comme dans les dispositifs institutionnels et les projets où il s'inscrit par sa visibilité numérique quantifiée à l'aide de multiples indicateurs, l'anthropologue ne peut prétendre à une distance substantielle avec les groupes sur lesquels il se propose de porter le regard sur la toile et qui eux-mêmes peuvent l'observer. Il en devient même obligatoirement un membre pour en comprendre les règles de fonctionnement interne et les modes de communication institués. Le subterfuge de créer son avatar où d'avancer sous pseudonyme n'est qu'une barrière illusoire à l'interpénétration des univers et comme l'a montré Wiki leaks il n'existe plus de mur sécuritaire absolument garanti dans les configurations contemporaines; quant aux identités multiples, phénomène typique de la Toile sur lequel la littérature abonde, elles seraient d'un piètre recours à l'anthropologue recherchant sa dernière tour d'ivoire. Cette nouvelle condition entièrement partagée avec les acteurs ciblés n'est pas identifiable avec les anciens modes d'immersion dans les groupes sociaux antérieurement étudiés: les retraits de l'anthropologue et son retour dans sa société, son univers coutumier étaient en effet partie prenante du parcours. Aujourd'hui, au contraire, à «la vie en double » que décrit si élégamment Marc Augé (2011), faite des voyages de l'anthropologue, s'est substituée une implication communicationnelle permanente, prenant appui sur un outil unique égalisant les partenaires des messages, quelle que soit la densité des rhizomes en jeu. La séparation est désormais impossible, l'ensemble des plages de communications communique et nul refuge ne s'offre à l'anthropologue comme à quiconque quelle que soit la population sur laquelle il se penche.

9 Quelles conséquences épistémologiques tirer de cette transformation profonde du réel qui démasque les stratégies de repli, évacue la sphère du privé, neutralise d'une certaine manière prévalences et hiérarchies dans la sphère globale de communication? Depuis déjà quelques décennies, l'anthropologue écrivait sous contrôle des populations l'utilisant pour leur reconnaissance, tels les peuples dits autochtones très actifs dans l'espace idéologique global de redéfinition du capitalisme. Que toutes les publications de l'anthropologue puissent être traduites et vues par les populations concernées ou non n'est pas un phénomène radicalement nouveau. La rupture épistémologique se joue en revanche au cœur même du positionnement de l'anthropologue, dont l'existence se voit tendanciellement banalisée malgré lui dans l'éventail présent des rôles possibles et interchangeables de l'autoentrepreneur au militant politique numérique en passant par le promoteur commercial de ses ouvrages, et ce d'autant plus que le métier ne sera plus bientôt adossé à des structures publiques de recherche mais intègrera les chaînes processuelles des contractualisations. Enfin, comme tout en chacun, l'anthropologue est affecté par l'affaiblissement et la désintégration de la distinction entre auteur et lecteur que met en œuvre l'inflation numérique (Duhelhi 2008) et sa fonction d'auteur devient très dépendante de sa compétence numérique. Plus que jamais l'anthropologue est donc enjoint à construire de toutes pièces, ex nihilo, une position dont la légitimité ne peut 
advenir que de la clarté de son objectif, exclusivement cognitif, l'affichage de finalités ne pouvant qu'opacifier sa tâche, dans les investigations mais aussi dans leurs restitutions écrites. En effet, la programmation d'une utilité éventuelle de ses recherches le positionne au milieu d'une multitude d'acteurs, présents comme lui dans la sphère globale de communication et avec lesquels il rentre immédiatement en concurrence, en débat, jouant à coup sûr perdant tant il est de fait démuni des armes médiatiques que ces derniers possèdent.

10 L'anthropologue ferait ainsi figure d'un funambule oxymorique, pleinement conscient des précipices communicationnels qui l'entourent et du fil étroit sur lequel il avance en tanguant. Ecartant les tentations d'identités agrégatives et polyphoniques, si courantes sur la toile, il s'en tiendrait à une solide épistémologie, épurée des scories du moment. Mais il serait de plus en plus attentif aux modes numériques de production des normes globales et à la participation ingénue et inventive des acteurs individuels et collectifs à leur légitimation, selon des logiques toujours singulières. Dans cette optique la singularité reste au cœur de la quête laborieuse de l'anthropologue dans un monde global qu'Internet chevauche de part en part. Corollairement les mutations extraordinaires des rapports de domination qu'Internet initie demandent à être analysées dans leurs amphibologies de plus en plus paroxystiques. En effet, d'un côté on discerne comment l'outil numérique peut ébranler et renverser les despotismes de toutes sortes, mais aussi les hiérarchies internes aux institutions, un des exemples les plus évident en étant les élèves interpellant sur facebook leurs professeurs devenus des «amis». De l'autre on perçoit un double processus conjoint de dépersonnalisation et de repersonnalisation de l'autorité, favorisant et/ou de façon alternative étouffant les rebellions. En outre des activistes attaquent frontalement les dominants, comme en Chine où traqués par une campagne numérique sauvage des fonctionnaires ont dû finalement être mis en prison. D'une manière générale après le communisme défunt, l'islamisme politique décapité, c'est bien le cyberterrorisme qui peut le plus violemment menacer le capitalisme actuel. Globalisé, financiarisé, le capitalisme dans sa forme présente semble appeler selon d'aucuns une "guerre civile numérique " (Jorion, 2011) le menant à son agonie. Cette dernière fait envisager par ailleurs pour les anthropologues une foule de terrains non défrichés, stimulant leur imagination et éclairant les angles noirs du présent.

YANG Wen, Self-expression and discourse on female sexuality: Online Sex discussion forums in Contemporary China, Master degree of philosophy, university of Hongkong, April 2009.

\section{BIBLIOGRAPHIE}

AUGÉ Marc (2011) : La vie en double, Payot

BADALOV Ulugbek (2011) : « Repli identitaire et imaginaire national chez les expatriés Kirghiztanais en france », Doctorat EHESS.

DING Yu, Transitions and New possibilities of sex work: Xiaojies' perception of work and way of life in the Pearl River Delta, PhD degree of philosophy, University of Hongkong, December 2008 
DOULHEIHI Milad (2008) : La grande conversion numérique, Le Seuil

GUO Wenjing (2011) : « Internet un outil de légitimation pour les homosexuels cantonais », in Enjeux épistémologiques et idéologiques de la globalisation pour les Sciences Sociales, B. Castelli, B. Hours, l'Harmattan.

HEAS Stéphane, Poutrain Véronique (2003) : « Les méthodes d'enquête qualitative sur Internet », Ethnographique, Org $\mathrm{n}^{\circ} 4$.

JORION Paul (2011) : La guerre civile numérique, Textuel.

PEI Yuxin, Born in the 70's: Sexuality of young women in contemporary Shanghai, PhD Degree of philosophy, University of Hongkong, december 2007

SELIM Monique (2011) : «L'importation des gender studies à Canton : usages personnels, collectifs, politiques ", in Enjeux épistémologiques et idéologiques de la globalisation pour les sciences sociales, B. Castelli , B. Hours, l'Harmattan.

USMANOVA Dilorom (2010) : Internet, nouvel espace pour les dissidents Ouzbékistanais (M2, EHESS).

\section{AUTEUR}

\section{MONIQUE SELIM}

Professeure d'anthropologie, Université Paris I. 